\title{
AMSS-Net: Audio Manipulation on User-Specified Sources with Textual Queries
}

\author{
Woosung Choi \\ Department of Computer Science, \\ Korea University \\ Seoul, Republic of Korea \\ ws_choi@korea.ac.kr
}

\author{
Minseok Kim \\ Department of Computer Science, \\ Korea University \\ Seoul, Republic of Korea \\ rlaalstjr47@korea.ac.kr
}

\author{
Marco A. Martínez Ramírez \\ Centre for Digital Music, \\ Queen Mary University of London \\ London, United Kingdom \\ m.a.martinezramirez@qmul.ac.uk
}

\author{
Jaehwa Chung \\ Department of Computer Science and \\ Engineering, \\ Korea National Open University \\ Seoul, Republic of Korea \\ jaehwachung@knou.ac.kr
}

\author{
Soonyoung Jung \\ Department of Computer Science, \\ Korea University \\ Seoul, Republic of Korea \\ jsy@korea.ac.kr
}

\begin{abstract}
This paper proposes a neural network that performs audio transformations to user-specified sources (e.g., vocals) of a given audio track according to a given description while preserving other sources not mentioned in the description. Audio Manipulation on a Specific Source (AMSS) is challenging because a sound object (i.e., a waveform sample or frequency bin) is 'transparent'; it usually carries information from multiple sources, in contrast to a pixel in an image. To address this challenging problem, we propose AMSS-Net, which extracts latent sources and selectively manipulates them while preserving irrelevant sources. We also propose an evaluation benchmark for several AMSS tasks, and we show that AMSS-Net outperforms baselines on several AMSS tasks via objective metrics and empirical verification.
\end{abstract}

\section{CCS CONCEPTS}

- Applied computing $\rightarrow$ Sound and music computing; • Computing methodologies $\rightarrow$ Neural networks.

\section{KEYWORDS}

audio manipulation, neural networks, text-guided

\section{ACM Reference Format:}

Woosung Choi, Minseok Kim, Marco A. Martínez Ramírez, Jaehwa Chung, and Soonyoung Jung. 2021. AMSS-Net: Audio Manipulation on User-Specified Sources with Textual Queries. In Proceedings of the 29th ACM International Conference on Multimedia (MM '21), October 20-24, 2021, Virtual Event, China. ACM, New York, NY, USA, 9 pages. https://doi.org/10.1145/3474085.3475323

\section{INTRODUCTION}

In recent days, social media applications have attracted many users to create, edit, and share their audio, audio-visual, or other types of

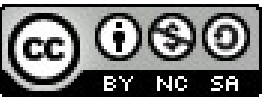

This work is licensed under a Creative Commons Attribution-NonCommercialShareAlike International 4.0 License.

MM '21, October 20-24, 2021, Virtual Event, China.

(C) 2021 Copyright held by the owner/author(s).

ACM ISBN 978-1-4503-8651-7/21/10.

https://doi.org/10.1145/3474085.3475323 multimedia content. However, it is usually hard for non-experts to manipulate them, especially when they want to edit only the desired objects. For image manipulation, fortunately, recently proposed methods such as image inpainting [36], style transfer [38], and textguided image manipulation $[10,12]$ enables non-expert users to edit the desired objects while leaving other contents intact. These machine learned-based methods can reduce human labor for image editing and enable non-experts to manipulate their image without prior knowledge of tools that are often complicated to use.

On the other hand, little attention has been given to machine learning methods for automatic audio editing. It is challenging to edit specific sound objects (e.g., decrease the volume of cicada buzzing noise) with limited tools in the given audio. Considering that audio editing usually requires expert knowledge of audio engineering or signal processing, we explore a deep learning approach in conjunction with textual queries to lessen audio editing difficulty.

This paper addresses Audio Manipulation on Specific Sources (AMSS), which aims to edit only desired objects that correspond to specific sources, such as vocals and drums, according to a given description while preserving the content of sources that are not mentioned in the description. We formally define AMSS and a structured query language for AMSS in $§ 2$. AMSS can be used for many applications such as video creation tools making audio editing easy for non-experts. For example, users can decrease the volume of drums by typing simple textual instructions instead of time-consuming interactions with digital audio workstations.

Although many machine learning approaches have been proposed for audio processing [3, 8, 13, 14, 18, 19, 23, 24, 28, 32, 33], to the best of our knowledge, there is no existing method that can directly address AMSS (see $§ 3$ ). This paper proposes a novel endto-end neural network that performs AMSS according to the given textual query. Designing a neural network for AMSS is straightforward if the sources of a given mixture track are observable. However, we assume that they are not observable because most audio data does not provide them in general. In the assumed environment, modeling AMSS is very challenging because a sound object (e.g., a sample in a wave, a frequency bin in a spectrogram) is 'transparent'([34]); a pixel in an image usually corresponds to only a single visual object, whereas a sound object carries information 


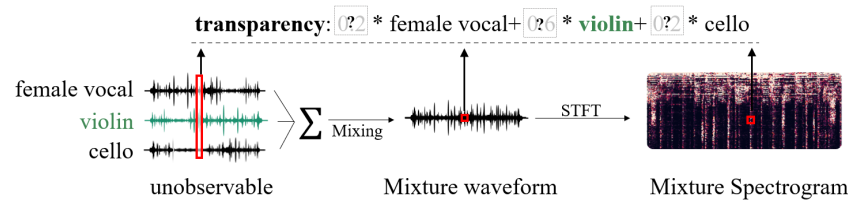

Figure 1: Sound objects are transparent

of multiple sources, as shown in Figure 1. Thus, we need different approaches for AMSS from the existing image manipulation techniques.

To address this challenge, we propose a neural network called AMSS-Net that extracts a feature map containing latent sources (see §5.4.1) from the given mixture audio and selectively manipulates them while preserving irrelevant latent sources. We describe the AMSS-Net architecture in $\S 5$.

Another challenge is that existing datasets cannot be directly used for supervised training AMSS-Net. If a training dataset of triples $\left\{\left(A^{(i)}, A^{\prime(i)}, S^{(i)}\right)\right\}_{i=1}^{N}$, where $S^{(i)}$ is an AMSS description, $A^{(i)}$ is a mixture, and $A^{\prime(i)}$ is the manipulated audio according to $S^{(i)}$ is provided, we can train a neural network net in a supervised manner by minimizing $\sum_{i=1}^{N} \operatorname{loss}\left(\operatorname{net}\left(A^{(i)}, S^{(i)}\right), A^{\prime(i)}\right)$, where loss is a distance metric such as $L_{2}$. Unfortunately, there were no datasets currently available that directly target AMSS. To address this issue, we propose a training framework for AMSS (see §4) that uses a 'source observable multi-track dataset' such as Musdb18 [22]. To generate an AMSS triple on-the-fly, we apply audio transformations onto specific sources of a given multi-track using common methods from Digital Signal Processing (DSP) libraries.

We summarize our contributions as follows:

- Our work is a pioneer study on selective audio manipulation.

- We propose a supervised training framework for AMSS based on source observable multi-track datasets and DSP libraries together with evaluation benchmarks for AMSS.

- We propose AMSS-Net, which performs multiple tasks and outperforms baselines on several tasks.

\section{TASK DEFINITION}

\subsection{Audio Manipulation on Specific Sources}

We define Audio Manipulation on Specific Sources (AMSS) as follows: for a given audio track $A$ and a given description $S$, AMSS aims to generate a manipulated audio track $A^{\prime}$ that semantically matches $S$ while preserving contents in $A$ that are not described in $S$. A contains multiple sources, and $S$ describes the desired audio transformation and the targets, which we want to manipulate. $S$ can be represented as a one-hot encoding or a textual query. In this paper, we assume that $S$ is a textual query written in the Audio Manipulation Language described in $§ 2.2$.

This paper focuses on modifying specified sources' sonic characteristics. This paper does not address complex manipulations such as distortion (see §6.7). Throughout the rest of the paper, we define an AMSS task to be a set of instructions dealing with the same manipulation method. Table 1 lists nine AMSS tasks we try to model in this paper. We also define an AMSS task class as a set of similar AMSS tasks.

\begin{tabular}{|c|c|l|}
\hline class & task & DSP operations \\
\hline \multirow{4}{*}{ volume control } & separate & masking the others \\
\cline { 2 - 3 } & mute & masking targets \\
\cline { 2 - 3 } & increase vol & re-scaling (increase) \\
\cline { 2 - 3 } & decrease vol & re-scaling (decrease) \\
\hline $\begin{array}{c}\text { volume control } \\
\text { (multi channel) }\end{array}$ & pan left & re-scaling (left $>$ mean > right) \\
\cline { 2 - 3 } filter & pan right & re-scaling (left $<$ mean < right) \\
\cline { 2 - 3 } & lowpasss & Low-pass Filter \\
\hline delay & highpass & High-pass Filter \\
\hline
\end{tabular}

Table 1: List of AMSS tasks modeled in this paper: (*) denotes reversed generation process (the line 5 in Algorithm 1)

\subsection{Audio Manipulation Language}

We assume that $S$ is given as a textual query, such as "apply light lowpass to drums". Textual querying enables us to naturally describe any pair of a transformation function and its target sources with detailed options. For example, we can control the level of audio effects by simply inserting adjectives such as light, medium, or heavy into the query. It also can provide easy extensibility to natural language interfaces, which will be addressed in future works.

To this end, we propose an Audio Manipulation Language based on a probabilistic Context-Free Grammar (CFG) [5] for AMSS. Due to the page limit, we only present a subset of production rules (i.e., Rules (1a)-(1f)) that define the query language's syntax for the filter class. The Full CFG is available online ${ }^{1}$.

$$
\begin{aligned}
& <\text { desc }>\rightarrow<\text { cls } s_{f}> \\
& <\text { cls }>\rightarrow \text { apply }<\text { opt }- \text { filter }>\text { to }<\text { srcs }> \\
& <\text { opt }- \text { filter }>\rightarrow<\text { opt }><\text { filter }>\mid<\text { filter }> \\
& <\text { opt }>\rightarrow \text { light } \mid \text { medium } \mid \text { heavy } \\
& <\text { filter }>\rightarrow \text { lowpass } \mid \text { highpass } \\
& <\operatorname{srcs}>\rightarrow \text { vocals } \mid \text { drums } \mid \text { bass } \mid \text { vocal, drums } \mid \ldots
\end{aligned}
$$

In the above rules, bold strings are terminal symbols, and strings enclosed in angle brackets are non-terminal symbols. Each rule is of the form $A \rightarrow \alpha|\beta| \ldots$, which means that $A$ can be replaced with $\alpha$ or $\beta$. In a CFG, we apply a rule to replace a single nonterminal symbol with one of the expressions. Starting from the first symbol $<$ desc $>$, we can generate a valid query string by recursively applying rules until there is no non-terminal symbol. For example, "apply medium lowpass to vocals, drums" can derived from < desc > by Rules (1a)-(1f). We can also produce "apply lowpass to vocals, drums" if we choose $<$ filter $>$ instead of $<o p t><$ filter $>$. Since we set a default option for lowpass level to be medium, those two queries have the same meaning.

Rules (1b)-(1e) are dependant on a AMSS task class, and Rule (1f) is dependant on a given multi-track audio. In this work, we use four AMSS task classes as shown in Table 1. Since we use in the experiment Musdb18[22] dataset of which track contains three named instruments (i.e., vocals, drums, bass), we set the right-hand side of Rule (1f) to have all the possible permutations.

\footnotetext{
${ }^{1}$ https://kuielab.github.io/AMSS-Net/aml.html
} 


\section{RELATED WORK}

Since the past decade, data-driven approaches for audio manipulation have been active research fields. Meanwhile, there were virtually no studies that directly addressed AMSS. An exception was an early algorithmic method [1], which proposed a framework that identifies and controls volumes of desired sources based on digital signal processing. Unlike [1], this paper presents a machine learning-based approach for various AMSS tasks as well as volume controls. We review the literature related to machine learning-based studies for audio manipulation.

Audio Effect Modeling: As described in [28], audio effects are used to modify perceptual attributes of the given audio signal, such as loudness, spatialization, and timbre. Recently, several methods $[13,14,23,28,33]$ have been proposed for audio effect modeling with deep neural networks. [23] proposed a convolutional network performing equalization. [13,14] proposed convolutional and recurrent networks for nonlinear audio effects with Long and Short-Term Memory [7], such as distortion and Leslie speaker cabinet. [28] presented an efficient neural network for modeling an analog dynamic range compressor enabling real-time operation on CPU.

All the methods above assume an audio input with a single source, while our method assumes a mixture of multiple sources as input. Also, they are task-specific (the trained model provides only one audio effect), while our approach can perform several manipulations with a single model.

Automatic Mixing: Audio mixing is a task that combines multitrack recordings into a single audio track. Before taking the sum of signals, mix engineers usually apply various audio signal processing techniques such as equalization and panning for a better hearing experience. Some approaches have attempted to automate audio mixing with deep neural networks. For example, [15] trained endto-end neural networks for automatic mixing of drums, where the audio waveform of individual drum recordings is the input of the model and the post-produced stereo mix is the output. [27] proposed networks based on temporal dilated convolutions to learn neural proxies of specific audio effects and train them jointly to perform audio mixing. These methods mainly focus on developing experttype mixing models that combine individual sources into a mixture track, regardless of user control or input for the desired type of transformation. On the other hand, AMSS-Net aims to manipulate user-specified sources in a desired manner, preserving others, where individual sources are not observable.

Unlike [15, 16], [17] assumed an environment where individual sources are not provided as input. They proposed an algorithmic framework that automatically remixes early jazz recordings, which are often perceived as irritating and disturbing from today's perspective. It first decomposes the given input into individual tracks by means of acoustic source separation algorithms and remixes them using automatic mixing algorithms. [19] proposed a replacement of the source separation and the mixing processes by deep neural networks. The environment assumed in [19] is the most similar to ours. However, [19] mainly focuses on remixing to change the audio mixing style, while this paper focuses on modifying specified sources in the desired manner.

Source Separation: Our work is also related to deep learningbased source separation methods [3, 4, 8, 11, 18, 24, 35]. While early methods separate either a single source $[4,8,35]$ or multiple sources once [11], conditioned source separation methods $[3,18,24]$ isolate the source specified by an input symbol. A conditioned source separation task can be viewed as an AMSS task where we want to simply mute all the unwanted sources. We propose a method that can perform several AMSS tasks as well as source separation tasks using a single model, and controlled with a textual query.

We can design an algorithmic framework for AMSS using an existing separation method as a preprocessor, similar to [17]. It can perform some AMSS tasks by separating the individual sources and applying the appropriate DSP functions to target sources. However, source separation methods also generate minor artifacts, which are not present in the original source. Although they are negligible in each source, they can be large enough to be perceived when we sum the separated results for AMSS. Instead, we propose an end-to-end neural network since our approach can model more complex transformations such as dereverberation, which cannot be easily modeled with DSP algorithms. Still, our model must have the ability to extract the target sources to be manipulated. Inspired by recent deep learning methods, we design a novel model that extracts latent sources, selectively manipulates them, aggregates them, and outputs a mixture that follows a textual query.

The concept of latent source has been introduced in recent source separation methods $[3,32]$. [32] have trained their model to separate the given input into a variable number of latent sources, which can be remixed to approximate the original mixture. By carefully taking the weighted sum of separated latent sources, we can extract the desired source, such as clean speech. [3] also use the concept of latent source for conditioned source separation. They proposed the Latent Source-Attentive Frequency Transformation (LaSAFT) method, which extracts the feature map for each latent source and takes the weighted sum of them by using an attention mechanism. We also analyze latent sources in AMSS-Net, where each latent source deals with a more detailed aspect of acoustic features than a symbolic-level source (e.g., 'vocals'). Similar to [3], we assume that a weighted sum of latent sources can represent a source, while [32] assumed that latent sources are independent. Unlike previous works, our approach is based on channel-level separation as described in §5.4.1. Each decoding block of AMSS-Net extracts latent source channels so that each channel can be correlated to a latent source.

\section{TRAINING FRAMEWORK FOR AMSS}

We propose a novel training framework that uses a multi-track dataset. It generates an AMSS triple $\left(A, A^{\prime}, S\right)$ on the fly by applying DSP library transformations or audio effects onto target sources of a given multi-track audio file. For instance, suppose that we have a randomly generated query string $S=$ 'apply lowpass to drums' using the CFG and a multi-track that consists of three sources, namely, a vocal track $a_{0}$, a bass track $a_{1}$, and a drum track $a_{2}$. Our training framework takes the linear sum (i.e., $A=\sum a_{j}$ ) to generate $A$ to generate corresponding input $A$. For the target audio $A^{\prime}$, it computes $\sum_{j \notin \tau} a_{j}+\sum_{j \in \tau} f\left(a_{j}\right)$, where $\tau$ and $f$ is the set of target sources and DSP function described in $S$, respectively. Our framework applies a Low-pass Filter (LPF) to $a_{2}$, and takes the sum for $A^{\prime}$ as follows: $A^{\prime}=a_{0}+a_{1}+\operatorname{LPF}\left(a_{2}\right)$. By doing so, it can generate an AMSS triple on-the-fly for a given description $S$. 


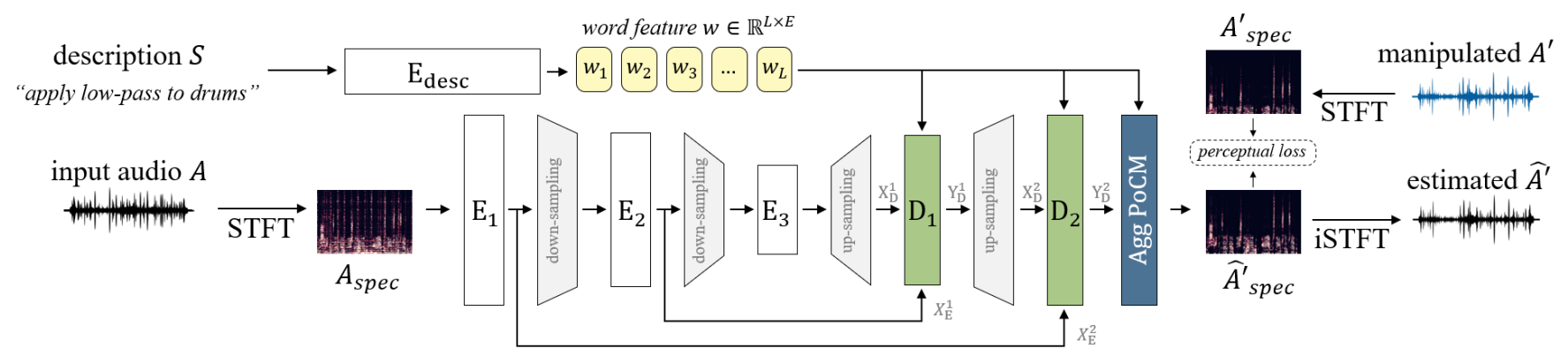

Figure 2: AMSS-Net Architecture

For audio restoration tasks such as dereverberation, we swap A and A'. For example, the framework applies reverb to $a_{2}$, takes the sum for $A^{\prime}=a_{0}+a_{1}+\operatorname{reverb}\left(a_{2}\right)$, and returns $\left(A^{\prime}, A, S\right)$ instead of $\left(A, A^{\prime}, S\right)$ for the description "remove reverb from drums."

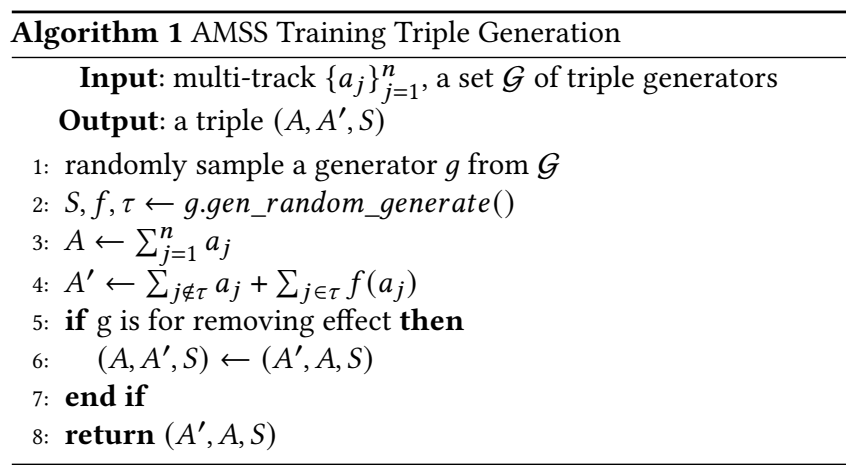

The training framework has a set $\mathcal{G}$ of triple generators. A generator $g \in \mathcal{G}$ has a subset of CFG for text query generation and the corresponding DSP function $f$, which is used for computing $A^{\prime} . g$ also has an indicator that describes whether $g$ is for applying or removing the effect. For a multi-track $\left\{A_{j}\right\}_{j=1}^{n}$ and a set $\mathcal{G}$ of triple generators, our framework generates an AMSS triple by using Algorithm 1, where $n$ denotes the number of sources.

\section{AMSS-NET ARCHITECTURE}

AMSS-Net takes as input an audio track $A$ with a text description $S$ and generates the manipulated audio track $\hat{A}^{\prime}$ as shown in Figure 2. It consists of two sub-networks, i.e., a Description Encoder $\mathrm{E}_{\text {desc }}$ and a Spectrogram Encoder-Decoder Network SEDN. $\mathrm{E}_{\text {desc }}$ extracts word features $w \in \mathbb{R}^{L \times E}$ from $S$, where $E$ denotes the dimension of the word features and $L$ denotes the number of words, to analyze the meaning of $S$. SEDN takes as input word features $w$ and the complex-valued spectrogram $A_{\text {spec }}$ of the input audio $A$. Conditioned on the word feature $w$, it estimates the complexvalued spectrogram ${\hat{A^{\prime}}}_{\text {spec }}$, from which $\hat{A}^{\prime}$ can be reconstructed using iSTFT. We train the AMSS-Net by minimizing the $L_{2}$ loss between the ground-truth spectrogram $A_{\text {spec }}^{\prime}$ of $A^{\prime}$ and $\hat{A}^{\prime}{ }_{\text {spec. }}$.

\subsection{Description Encoder}

The description encoder $\mathrm{E}_{\text {desc }}$ encodes the given text description $S$ written in the Audio Manipulation Language (§2.2) to word features $w \in \mathbb{R}^{L \times E}$, where we denote the dimension of each word feature by $E$ and the number of words in $S$ by $L$. It embeds each word to a dense vector using a word embedding layer and then encodes the embedded description using a bidirectional Recurrent Neural Network (Bi-RNN) [25]. We use pre-trained word embeddings such as GloVe[20] to initialize the weight of the embedding layer since they were trained to capture the syntactic and semantic meaning of words.

\subsection{Spectrogram Encoder-Decoder Network}

The Spectrogram Encoder-Decoder Network SEDN estimates the complex-valued spectrogram $\hat{A}^{\prime}{ }_{\text {spec }}$, conditioned on the extracted word features $w \in \mathbb{R}^{L \times E}$. It is an encoder-decoder network that has the same number of down-sampling layers and up-sampling layers as depicted in Figure 2. It extracts down-sampled representations from $A_{\text {spec }}$ in the encoding phase and generates up-sampled representations in the decoding phase. The output of the last decoding block is fed to the Aggregate PoCM (see §5.5) that generates the output ${\hat{A^{\prime}}}_{\text {spec. }}$.

As illustrated in Figure 2, it has direct connections between the encoding blocks and their counterpart decoding blocks, which help decoding blocks recover fine-grained details of the target. Instead of concatenation or summation commonly used in several U-Netbased architectures [3, 4, 8, 18], we propose a Channel-wise Skip Attention (CSA) mechanism (§5.4.4) that attentively aggregates latent source channels to reconstruct the original channels.

As shown in Figure 2, SEDN consists of several components: encoding blocks, down/up-sampling layers, decoding blocks, and an Aggregate PoCM. We use strided convolutions and transposed convolutions for down-sampling and up-sampling, respectively. We introduce other componets in $§ 5.3, \S 5.4$, and $§ 5.5$.

\subsection{Spectrogram Encoding Block}

$S E D N$ uses multiple encoding blocks in the encoding phase to capture common sonic properties residing in the input spectrogram. The $k^{t h}$ encoding block $E_{k}$ transforms an input spectrogram-like tensors into an equally-sized tensor. We adopt the encoding block called TFC-TDF proposed in [4], which applies densely-connected 2-d convolutions to the given spectrogram-like representations followed by a fully connected layer that enhances features of frequency patterns observed in the frequency axis. 


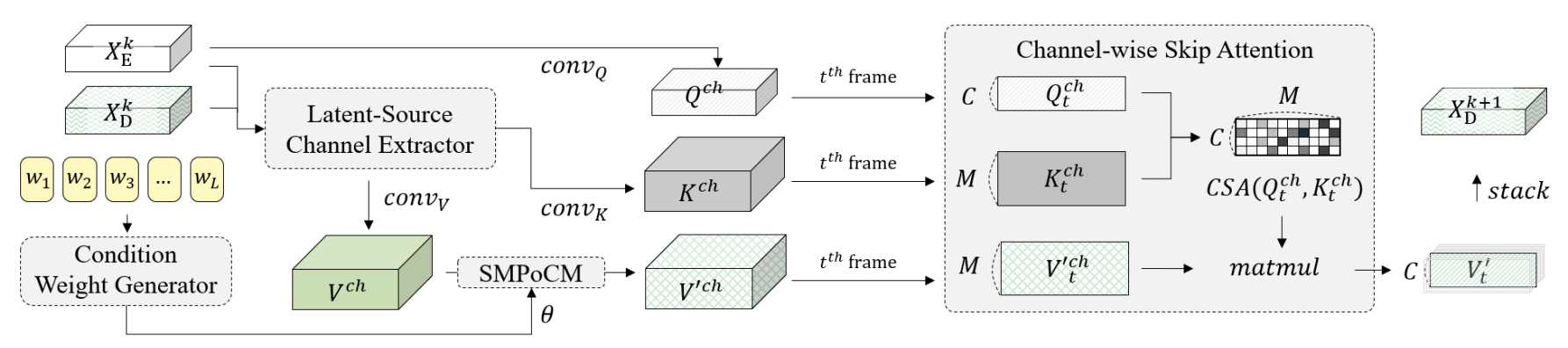

Figure 3: $k^{\text {th }}$ decoding block $\left(D_{k}\right)$

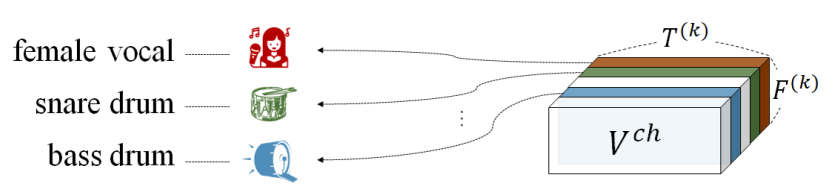

Figure 4: Conceptual View of Latent Source Channels

\subsection{Spectrogram Decoding Block}

In the decoding phase, SEDN uses multiple decoding blocks. Each decoding block first extracts a feature map in which each channel corresponds to a specific latent source. It selectively manipulates them conditioned on the AMSS description and aggregates channels using a channel-wise attention mechanism to minimize information loss during channel reconstruction.

As shown in Figure 3, the $k^{\text {th }}$ decoding block $D_{k}$ takes three inputs: (1) $X_{D}^{k}$ : features from the previous decoding block, (2) $X_{E}^{k}$ : features from the skip connection and (3) $w \in \mathbb{R}^{L \times E}$ : word features. The first block takes the up-sampled features from the encoder instead because it has no previous decoding block.

Each decoding block consists of four components: Latent Source Channel (LSC) Extractor, Condition weight generator, Selective Manipulation via PoCMs (SMPoCM), and Channel-wise Skip Attention (CSA). We illustrate the overall workflow in Figure 3.

5.4.1 Latent Source Channel Extractor. We assume that we can learn representations of latent sources that deal with more detailed acoustic features than symbolic-level sources. The Latent Source Channel (LSC) extractor aims to generate a feature map $V^{\text {ch }}$, in which each channel deals with a latent source. Figure 4 visualizes the conceptual view of latent source channels. Each channel is a spectrogram-like representation of size $T^{(k)} \times F^{(k)}$ dealing with a specific latent source. For example, the blue channel in Figure 4 deals with the acoustic features observed in the bass drum. We can also generate an audio track from a single latent source channel. In $\S 6.4$, we visualize and discuss results of the audio generation.

The LSC extractor takes $X_{E}^{k}, X_{D}^{k} \in \mathbb{R}^{C \times T^{(k)} \times F^{(k)}}$ as input and extracts feature maps with $M$ latent source channels, where $C$ refers to the number of channels, $T^{(k)} \times F^{(k)}$ refers to the shape of the spectrogram-like features, and $M$ denotes the number of latent sources. It first concatenates $X_{E}^{k}$ and $X_{D}^{k}$ to obtain $\left[X_{E}^{k} ; X_{D}^{k}\right] \in$
$\mathbb{R}^{2 C \times T^{(k)} \times F^{(k)}}$, and applies a TFC-TDF block [4] to $\left[X_{E}^{k} ; X_{D}^{k}\right]$ to obtain acoustic features $X^{k}$ for latent source separation.

To extract a feature map $V^{c h} \in \mathbb{R}^{M \times T^{(k)} \times F^{(k)}}$ with $M$ latent source channels, it applies a $1 \times 1$ convolution to $X^{k}$. We denote this convolution as conv $v_{V}$ since its role is a value generator in the context of the channel-wise skip attention mechanism §5.4.4 as shown in Figure 3. Similar to $\operatorname{conv}_{V}$, we apply another $1 \times 1$ convolution called $\operatorname{conv}_{K}$ to $X^{k}$, of which role is a key generator, to obtain $K^{c h}$. By the guide of the channel-wise skip attention mechanism, the LSC extractor is expected to extract latent source channels from the mixture features so that each channel deals with a latent source.

5.4.2 Selective Manipulation via PoCMs. Since we have to manipulate specific features while preserving other features, selective manipulation requires a more sophisticated modulation than existing methods such as Feature-wise Linear Modulation (FiLM) [21], or Point-wise Convolutional Modulation (PoCM) [3].

We propose SMPoCM to manipulate features for the given AMSS task selectively. As shown in Figure 3, it takes as input $V^{c h}$ and condition parameters $\theta=\left(\theta_{s}, \theta_{m}, \theta_{i}\right)$, generated by the LSC extractor and the condition weight generator, respectively. It outputs $V^{\prime c h} \in \mathbb{R}^{M \times T^{(k)} \times F^{(k)}}$, a selectively manipulated feature.

Inspired by Long Short-term Memory (LSTM) [7], the SMPoCM uses three different PoCMs: (1) a selective gate PoCM with $\theta_{s}$ to determine how much we should manipulate each latent source channel, (2) a manipulation PoCM with $\theta_{m}$ to manipulate specific features, and (3) an input gate PoCM with $\theta_{i}$ to determine how much we should emit the manipulated features.

Before defining SMPoCM formally, we summarize the behavior of PoCM as follows: a PoCM is a point-wise convolution (i.e., $1 \times 1$ convolution) of which the condition weight generator provides parameters. We now give a definition of SMPoCM as follows: $\operatorname{SMPoCM}(X \mid \theta)=i \odot \tanh \left(\operatorname{PoCM}\left(s \odot X, \theta_{m}\right)\right)+(1-s) \odot X$, where $s$ is defined as $\sigma\left(\operatorname{PoCM}\left(X, \theta_{s}\right)\right), i$ is defined as $\sigma\left(\operatorname{PoCM}\left(X, \theta_{i}\right)\right)$, $\odot$ is Hadamard product, and $\sigma$ is a sigmoid function. SMPoCM naturally models the selective modulation required for modeling AMSS tasks. For example, if $i^{t h}$ source should be preserved for the given input, then the $i^{\text {th }}$ channel of $s$ would be trained to have near-zero values.

5.4.3 Condition Weight Generator. Given $w \in \mathbb{R}^{L \times E}$, the condition weight generator generates parameters $\theta=\left(\theta_{s}, \theta_{m}, \theta_{i}\right)$. We exploit the attention mechanism to determine which word should be attended to $\theta_{s}, \theta_{m}$, and $\theta_{i}$ respectively. 
The weight generator has a learnable matrix $\Theta \in \mathbb{R}^{3 \times d_{k}}$, where we denote the cardinality of each PoCM task embedding by $d_{k}$. It also has two linear layers linear $_{k}$ and linear $v_{v}$ that embed $w$ to $w_{k e y} \in \mathbb{R}^{L \times d_{k}}$ and $w_{\text {value }} \in \mathbb{R}^{L \times d_{k}}$ respectively. To determine which word we should attend for each task, we compute the scaled attention [30] as follows: $\alpha^{w g}=\operatorname{softmax}\left(\frac{\Theta w_{k e y}}{\sqrt{d_{k}}}\right) w_{\text {value }}^{T}$. Finally, it generates $\theta_{s}, \theta_{m}$, and $\theta_{i}$ as follows: $\theta_{s}=\operatorname{linear}_{s}\left(\alpha^{w g}[0,:]\right)$, $\theta_{s}=\operatorname{linear}_{m}\left(\alpha^{w g}[1,:]\right)$, and $\theta_{s}=\operatorname{linear}_{i}\left(\alpha^{w g}[2,:]\right)$, where linear ${ }_{s}$, linear $_{m}$, and linear $i$ are fully-connected layers.

5.4.4 Channel-wise Skip Attention. Inspired by skip attention [37] and [2], we propose a Channel-wise Skip Attention (CSA) mechanism. It attentively aggregates $M$ latent source channels to restore the number of channels to the same as the input (i.e., $C$ ). The goal of CSA is to minimize information loss during channel reconstruction to preserve other features that are irrelevant to the description.

Figure 3 overviews the workflow required to prepare the query feature $Q^{c h}$, key feature $K^{c h}$, and the value feature $V^{\prime c h}$. To obtain $Q^{c h} \in \mathbb{R}^{C \times T^{(k)} \times F^{(k)}}$, we apply conv $Q$, a $1 \times 1$ convolution to $X_{E}^{k}$.

For each frame, CSA aims to capture channel-to-channel dependencies between $X_{E}^{k}$ that encodes the original acoustic features of $A$ and the feature map $K^{c h}$ of isolated latent sources obtained by the LSC extractor. It is worth noting that we use $K^{c h}$ for computing attention matrix instead of $V^{\prime c h}$ since $V^{\prime c h}$, which SMPoCM modulated, no longer has the same information as $X_{E}^{k}$.

For $Q_{t}^{c h}=Q^{c h}[:, t,:]$ and $K_{t}^{c h}=K^{c h}[:, t,:]$, we compute the scaled dot product attention matrix [30] as follows:

$$
C S A\left(Q_{t}^{c h}, K_{t}^{c h}\right)=\operatorname{softmax}\left(\frac{Q_{t}^{c h}\left(K_{t}^{c h}\right)^{T}}{\sqrt{F^{(k)}}}\right)
$$

The attention weight $C S A\left(Q_{t}^{c h}, K_{t}^{c h}\right)_{i, j}$ represents the correlation between the $i^{\text {th }}$ channel of the original audio features and the $j^{\text {th }}$ latent source channel of the decoded audio features. Finally, we can obtain the decoding block's output $y_{D}^{k}$, where $y_{D}^{k}[:, t,:]$ is defined as $\operatorname{CSA}\left(Q_{t}^{c h}, K_{t}^{c h}\right) V^{\prime c h}[:, t,:]$.

\subsection{Aggregate PoCM}

The Aggregate Pocm is similar to the SMPoCM other than two key differences. First, the Aggregate PoCM only has one PoCM that is not followed by any activation. Second, the Aggregate PoCM reduces the number of channels from $C$ to 4 (i.e., the number of channels of the two-channeled complex-valued spectrogram) while the SMPoCM's input and output have the same number of channels.

\section{EXPERIMENTS}

\subsection{Experiment Setup}

We evaluate our model qualitatively and quantitatively on various AMSS tasks described in Table 1 using the Musdb18 dataset. We compare its performance with baselines.

6.1.1 Training Framework. Musdb18 [22] contains 86 tracks for training, 14 tracks for validation, and 50 tracks for the test. Each track is stereo, sampled at $44100 \mathrm{~Hz}$, and each item consists of the

\begin{tabular}{|c|c|c|c|c|c|}
\hline model & vocals & drums & bass & other & AVG \\
\hline Meta-TasNet & 6.40 & 5.91 & 5.58 & 4.19 & 5.52 \\
\hline LaSAFT-GPoCM-Net $_{12}$ & 7.33 & 5.68 & 5.63 & 4.87 & 5.88 \\
\hline LaSAFT-GPoCM-Net $_{11}$ & 6.96 & 5.84 & 5.24 & 4.54 & 5.64 \\
\hline AMSS-Net $_{\text {separate }}$ & $\begin{array}{c}6.78 \\
\pm .12\end{array}$ & $\begin{array}{r}5.92 \\
\pm .03\end{array}$ & $\begin{array}{r}5.10 \\
\pm .06\end{array}$ & $\begin{array}{c}4.51 \\
\pm .10\end{array}$ & $\begin{array}{r}5.58 \\
\pm .90\end{array}$ \\
\hline AMSS-Net & $\begin{array}{c}\mathbf{6 . 3 4} \\
\pm .016 \\
\end{array}$ & $\begin{array}{r}5.53 \\
\pm .07 \\
\end{array}$ & \begin{tabular}{|l|}
4.33 \\
\pm .13 \\
\end{tabular} & $\begin{array}{r}3.99 \\
\pm .07 \\
\end{array}$ & $\begin{array}{r}5.05 \\
\pm .99 \\
\end{array}$ \\
\hline w/o CSA & $\begin{array}{c}6.03 \\
\pm .24 \\
\end{array}$ & $\begin{array}{c}5.53 \\
\pm .12 \\
\end{array}$ & $\begin{array}{r}4.22 \\
\pm .09 \\
\end{array}$ & $\begin{array}{c}3.73 \\
\pm .23 \\
\end{array}$ & $\begin{array}{r}4.88 \\
\pm .99 \\
\end{array}$ \\
\hline w/o SMPoCM & $\begin{array}{c}6.08 \\
\pm .25\end{array}$ & $\begin{array}{r}\mathbf{5 . 6 3} \\
\pm .08\end{array}$ & $\begin{array}{r}4.28 \\
\pm .19\end{array}$ & $\begin{array}{c}3.76 \\
\pm .04\end{array}$ & $\begin{array}{r}4.94 \\
\pm .99\end{array}$ \\
\hline
\end{tabular}

Table 2: A comparison SDR performance. LaSAFT-GPoCMNet $x$ uses n_FFT of $2^{x}$

mixture and four sources: vocals, drums, bass, and other. We implemented the training framework based on Musdb18 and pysndf ${ }^{2}$, a Python DSP library. We train all models based on this framework with 9 AMSS tasks listed in Table 1. For each task, we implement triple generators based on the Audio Manipulation Language (§2.2) and Musdb18. We exclude 'other' since it is not a single instrument. With the set $\mathcal{G}$ of triple generators and randomly generated multitracks obtained by data augmentation [29], we generate AMSS triples using Algorithm 1 for training.

6.1.2 Training Environment. We train models using Adam [9] with learning rate $l r \in[0.0001,0.001]$. Starting with a learning rate $l r$, we halved $l r$ and restarted training when the current $l r$ seemed to be too high. Each model is trained to minimize the $L_{2}$ loss between the ground-truth and estimated spectrograms. For validation, we use the $L_{1}$ loss of target and estimated signals. It takes about two weeks to converge when we train models with a single $2080 \mathrm{Ti}$ GPU.

\subsection{Model Configurations}

To validate the effectiveness of AMSS-Net, we compared it with the two baselines. One does not use CSA (AMSS-Net w/o CSA), and the other does not use SMPoCM in decoding blocks (AMSS-Net w/o SM). The baseline without CSA uses an LSC extractor with LaSAFT block [3] instead of TFC-TDF [4] to compensate the absence of CSA. The model without SMPoCM uses a PoCM with tanh in its decoding block. An AMSS-Net has about 4.3M, a baseline w/o CSA has about 4.9M, and a baseline w/o SMPoCM has about 2.4M.

For hyper-parameter setting, we use a similar configuration of models with an n_FFT of 2048 in [3]. Every model has three encoding blocks, two decoding blocks, and an Aggregate PoCM block. We assume that there are eight latent sources (i.e., $M=8$ ). We also adopt the multi-head attention mechanism [30] for CSA, where we set the number of heads to 6 . The STFT parameter of each model is as follows: an n_FFT of 2048 and a hop size of 1024.

\subsection{Quantitative Analysis}

6.3.1 Evaluation of separate and mute tasks (Table 2). To evaluate separate and mute tasks, we use Source-to-Distortion (SDR) [31]

\footnotetext{
${ }^{2}$ https://pypi.org/project/pysndfx/
} 


\begin{tabular}{|c|c|c|c|c|c|c|c|c|c|c|c|c|}
\hline & \multicolumn{3}{|c|}{ pan left } & \multicolumn{3}{|c|}{ pan right } & \multicolumn{3}{|c|}{ decrease volume } & \multicolumn{3}{|c|}{ increase volume } \\
\hline & voc & drum & bass & voc & drum & bass & voc & drum & bass & voc & drum & bass \\
\hline reference loss & 4.62 & 8.20 & 2.18 & 4.66 & 8.30 & 2.13 & 4.06 & 6.99 & 2.00 & 6.68 & 9.70 & 3.48 \\
\hline \multirow{2}{*}{ AMSS-Net } & 3.32 & 4.81 & 2.11 & 3.34 & 4.85 & 2.11 & 2.72 & 3.78 & 1.93 & 3.49 & 4.36 & 2.9 \\
\hline & \pm 0.19 & \pm 0.16 & \pm 0.13 & \pm 0.19 & \pm 0.19 & \pm 0.17 & \pm 0.23 & \pm 0.18 & \pm 0.13 & \pm 0.2 & \pm 0.18 & \pm 0.19 \\
\hline \multirow{2}{*}{ w/o CSA } & 3.65 & 5.46 & 2.61 & 3.63 & 5.53 & 2.58 & 3 & 4.26 & 2.32 & 4.47 & 5.11 & 3.66 \\
\hline & \pm 0.17 & \pm 0.2 & \pm 0.33 & \pm 0.11 & \pm 0.23 & \pm 0.3 & \pm 0.13 & \pm 0.14 & \pm 0.33 & \pm 0.12 & \pm 0.18 & \pm 0.4 \\
\hline \multirow{4}{*}{ w/o SMPoCM } & 4.34 & 5.51 & 3.23 & 4.23 & 5.5 & 3.19 & 3.55 & 4.41 & 2.92 & 4.16 & 5.2 & 3.4 \\
\hline & \pm 0.2 & \pm 0.16 & \pm 0.19 & \pm 0.18 & \pm 0.11 & \pm 0.17 & \pm 0.16 & \pm 0.16 & \pm 0.17 & \pm 0.14 & \pm 0.15 & \pm 0.17 \\
\hline & \multicolumn{3}{|c|}{ lowpass filter } & \multicolumn{3}{|c|}{ highpass filter } & \multicolumn{3}{|c|}{ dereverberation } & \multicolumn{3}{|c|}{ mean } \\
\hline & voc & drum & bass & voc & drum & bass & voc & drum & bass & voc & drum & bass \\
\hline reference loss & 9.02 & 16.27 & 1.12 & 6.72 & 8.22 & 4.74 & 9.43 & 11.07 & 5.61 & 6.46 & 9.82 & 3.04 \\
\hline \multirow{2}{*}{ AMSS-Net } & 7.32 & 10.92 & 2.65 & 5.65 & 6.9 & 3.8 & 6.12 & 6.72 & 4.18 & 4.57 & 6.05 & 2.81 \\
\hline & \pm 0.19 & \pm 0.15 & \pm 0.09 & \pm 0.28 & \pm 0.3 & \pm 0.11 & \pm 0.29 & \pm 0.23 & \pm 0.24 & \pm 1.70 & \pm 2.33 & \pm 0.85 \\
\hline \multirow{2}{*}{ w/o CSA } & 7.44 & 12.37 & 3.15 & 5.49 & 7.41 & 4.23 & 6.18 & 7.08 & 4.28 & 4.84 & 6.75 & 3.26 \\
\hline & \pm 0.07 & \pm 0.5 & \pm 0.21 & \pm 0.25 & \pm 0.67 & \pm 0.4 & \pm 0.12 & \pm 0.07 & \pm 0.1 & \pm 1.53 & \pm 2.60 & \pm 0.83 \\
\hline \multirow{2}{*}{ w/o SMPoCM } & 8.11 & 12.06 & 3.67 & 6.81 & 7.84 & 4.85 & 6.68 & 7.17 & 4.68 & 5.41 & 6.81 & 3.71 \\
\hline & \pm 0.1 & \pm 0.31 & \pm 0.23 & \pm 0.21 & \pm 0.24 & \pm 0.42 & \pm 0.12 & \pm 0.24 & \pm 0.05 & \pm 1.67 & \pm 2.47 & \pm 0.75 \\
\hline
\end{tabular}

Table 3: A RMSE-MFCC Comparison of our models with baselines, over 7 AMSS tasks applied to vocals, drums, and bass

metric by using the official tool ${ }^{3}$. Musdb18 tasks are separating vocals, drums, bass, and other, which corresponds to the following AMSS: 'separate vocals,' 'separate drums,' 'separate bass,' and 'mute vocals, drums, bass.' Following the official guideline, we report the median SDR value over all the test tracks for each run and report the mean SDR over three runs (with a different random seed). Table 2 summarizes the result, where AMSS-Net shows comparable or and slightly inferior performance compared to conditioned separation models, namely, Meta-TasNet [24] and LaSAFT-GPoCMNets. AMSS-Net outperforms the baselines for all sources except for drums, where the gap is not significant. It is worth noting that ours can perform other AMSS tasks and show promising results on source separation tasks. If we train AMSS-Net solely for Musdb18 tasks (AMSS-Net separate $_{\text {) }}$, then it shows comparable performance to LaSAFT-GPoCM-Net 11 whose n_FFT is the same as ours.

6.3.2 Evaluation of other AMSS tasks (Table 3). Since there is no reference evaluation metric for AMSS, we propose the new evaluation benchmark. The benchmark script is available online ${ }^{4}$. For evaluation metric, we extract Mel-frequency Cepstral coefficients (MFCC) for $A^{\prime}$ and $\hat{A}^{\prime}$, and compute the Root Mean Square Error (RMSE) of them, since MFCC approximates the human perception of the given track. We refer to this metric as RMSE-MFCC. We report the mean RMSE-MFCC value over all the test set tracks for each run and report the mean RMSE-MFCC over three runs.

Table 3 summarizes the results, where reference loss is the RMSEMFCC of $A$ and $A^{\prime}$. Reference loss provides information about the amount of manipulation needed for each AMSS task. As described in Table 3, AMSS-Net outperforms all the AMSS task but a task of "highpass filter to vocals". Significantly, the model without SMPoCM is inferior to AMSS-Net for every task. It indicates that SMPoCM significantly contributes to improving the quality of AMSS results. CSA also improves the performance of AMSS-Net for most of the

\footnotetext{
${ }^{3}$ https://github.com/sigsep/sigsep-mus-eval

${ }^{4}$ https://github.com/kuielab/AMSS-Net/blob/main/task2_eval.py
}

AMSS tasks. CSA might degrade the performance for more difficult AMSS tasks by forcing the model to over-correlate the latent source channels with the mixture channels. However, it reduces artifacts created during progressive manipulation as described in $\S 6.5$.

\subsection{Latent Source Channels}

As mentioned in $§ 5.4 .1$, we design AMSS-Net to perform latent source-level analysis. Such analysis enables AMSS-Net to perform delicate manipulation for the given AMSS task. To verify that AMSSNet our decoding blocks can extract a feature map in which each channel corresponds to a specific latent source, we generate an audio track from a single latent source channel. We mask all channels in the manipulated feature map $V^{\prime c h}$ except for a single latent source channel and fed it to the remaining sub-networks to generate the audio track during the last decoding block as shown in Figure 5. Figure 5 reminds us the conceptual view of the latent source in Figure 4. When we play the audio of Figure 5 (b), the result sounds similar to the low-frequency band of drums (i.e., kick drum). However, a latent source channel does not always contain a single class of instruments as shown in Figure 5 (d). Generated samples are available online. ${ }^{5}$

\subsection{Progressive Manipulation}

We can repeatedly apply the proposed method to manipulated audio tracks, which is also known as Progressive Manipulation used in conversational systems [12]. Figure 6 shows an example of progressive manipulation.

Neural networks sometimes suffer from artifacts [31], which are not present in the original source. Although they sound negligible after a single manipulation task, they can be large enough to be perceived after progressively applying several. To investigate artifacts created by progressive manipulation, we apply the same AMSS task "apply highpass to drums" to a track in a progressive

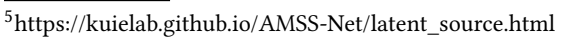




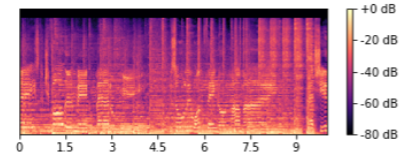

(a) original

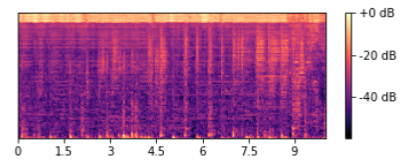

(c) high hat

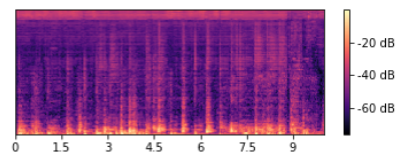

(b) kick drum

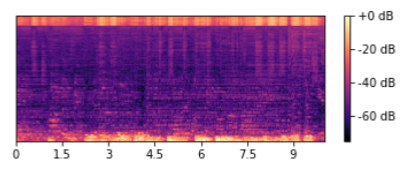

(d) non-percussion
Figure 5: Mel-Spectrogram of single latent-source channel

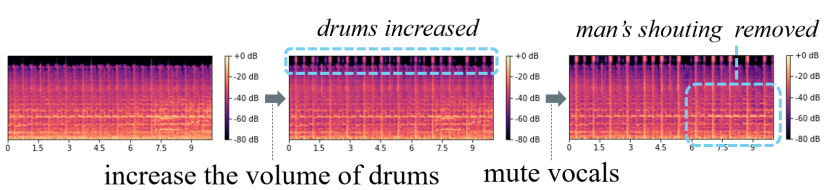

Figure 6: An Example of Progressive Manipulation

manner. Figure (a) shows the Mel-spectrogram of the ground-truth target. We can observe blurred areas in the high-frequency range in Mel-spectrograms of Figure 7 (c) and (d). Compared to them, Figure 7 (b) is more similar to the ground-truth target. Via hearing test, we observed perceivable artifacts in the results of baselines. Our AMSS-Net contains minor artifacts compare to them because each decoding block of AMSS-Net has a CSA mechanism, a unique structure that prevents unwanted noise generated by intermediate manipulated features. Generated samples are available online ${ }^{6}$. (a) ground-truth target

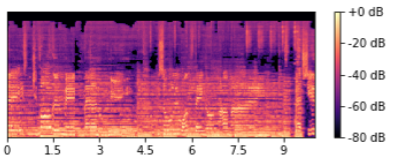

(c) w/o CSA

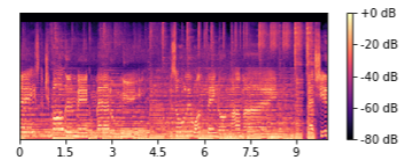

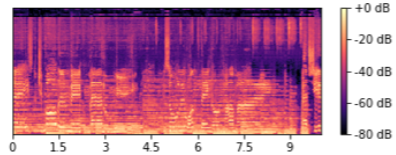

(b) AMSS-Net

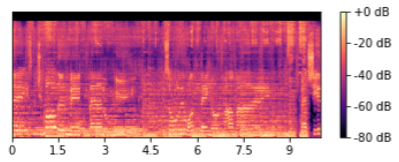

(d) w/o SMPoCM
Figure 7: Mel-Spectrogram Comparison after applying 20 times of 'apply highpass to drums' in a progressive manner

\subsection{Controlling the level of audio effects}

As mentioned in $\$ 2.2$, we can train AMSS-Net to perform more detailed AMSS such as "apply heavy lowpass to vocals". As shown in Figure 8, users can control the level of audio effects by simply injecting adverbs instead of a laborious search for an appropriate parameter configuration. Generated samples are available online ${ }^{7}$.

${ }^{6}$ https://kuielab.github.io/AMSS-Net/progresive.html

${ }^{7}$ https://kuielab.github.io/AMSS-Net/control_level.html

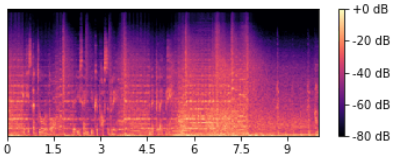

(a) original

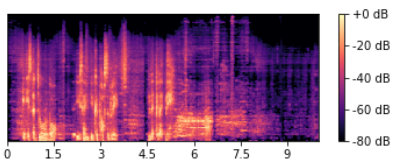

(c) separate vocals

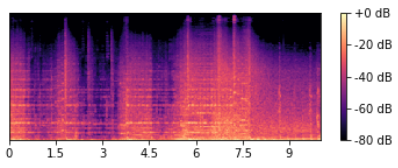

(e) mute vocals

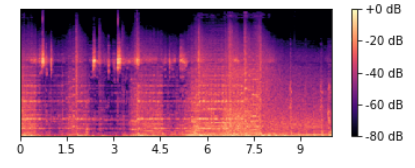

(b) heavy highpass to vocals

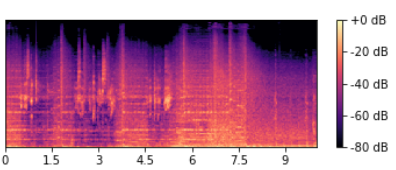

(d) medium highpass to vocals

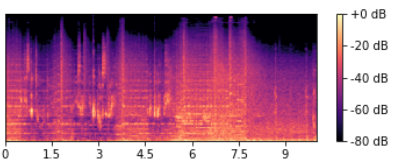

(f) light highpass to vocals
Figure 8: Controlling the level of highpass with adjectives

\subsection{Discussion}

AMSS-Net shows promising results on several AMSS tasks. AMSSNet can also be trained with a more complicated AMSS training dataset based on a realistic audio mixing dataset such as IDMT-SMTAudio-Effects dataset [26]. However, this study is limited to model relatively simpler AMSS tasks. One can extend our work to provide more complex AMSS tasks such as distortion and reverberation. Each AMSS task in this paper only deals with a single type of manipulations, but one can also extend our work to provide multiple types of tasks such as "apply reverb to vocals and apply lowpass to drums" at once. Also, our work is easily extendable to support a more user-friendly interface. For example, adopting unsupervised training frameworks such as Mixture of Mixture (MoM) [32] to train AMSS on annotated audio datasets such as clotho[6] might enable a natural language query interface.

\section{CONCLUSION}

This paper defines a novel task called AMSS. We propose AMSS-Net, which generates feature maps in which each channel deals with a latent source and selectively manipulates them while preserving irrelevant features. It can perform several AMSS tasks, unlike previous models such as LaSAFT-GPoCM-Net. The experimental results show that AMSS-Net outperforms baselines on several tasks. Future work will extend it to provide more complex AMSS tasks such as distortion and reverberation by adopting state-of-the-art methods such as Generative Adversarial Networks (GAN).

\section{ACKNOWLEDGMENTS}

This work was supported by the National Research Foundation of Korea (NRF) grant funded by the Korea government(MSIT)(No. 2020R1A2C1012624, 2021R1A2C2011452). Also, This research was supported by Basic Science Research Program through the National Research Foundation of Korea(NRF) funded by the Ministry of Education(No. 2019R1A6A3A13095526). 


\section{REFERENCES}

[1] Carlos Avendano. 2003. Frequency-domain source identification and manipulation in stereo mixes for enhancement, suppression and re-panning applications. In 2003 IEEE Workshop on Applications of Signal Processing to Audio and Acoustics (IEEE Cat. No. 03TH8684). IEEE, 55-58.

[2] Long Chen, Hanwang Zhang, Jun Xiao, Liqiang Nie, Jian Shao, Wei Liu, and TatSeng Chua. 2017. Sca-cnn: Spatial and channel-wise attention in convolutional networks for image captioning. In Proceedings of the IEEE conference on computer vision and pattern recognition. 5659-5667.

[3] Woosung Choi, Minseok Kim, Jaehwa Chung, and Soonyoung Jung. 2020. LaSAFT: Latent Source Attentive Frequency Transformation for Conditioned Source Separation. arXiv preprint arXiv:2010.11631 (2020).

[4] Woosung Choi, Minseok Kim, Jaehwa Chung, Daewon Lee, and Soonyoung Jung. 2020. Investigating u-nets with various intermediate blocks for spectrogrambased singing voice separation. In Proceedings of the 21th International Society for Music Information Retrieval Conference.

[5] N. Chomsky. 1956. Three models for the description of language. IRE Transactions on Information Theory 2, 3 (1956), 113-124. https://doi.org/10.1109/TIT.1956. 1056813

[6] Konstantinos Drossos, Samuel Lipping, and Tuomas Virtanen. 2020. Clotho: An audio captioning dataset. In ICASSP 2020-2020 IEEE International Conference on Acoustics, Speech and Signal Processing (ICASSP). IEEE, 736-740.

[7] Sepp Hochreiter and Jürgen Schmidhuber. 1997. Long Short-Term Memory. Neural Computation 9, 8 (1997), 1735-1780.

[8] Andreas Jansson, Eric Humphrey, Nicola Montecchio, Rachel Bittner, Aparna Kumar, and Tillman Weyde. 2017. Singing voice separation with deep u-net convolutional networks. In 18th International Society for Music Information Retrieval Conference. 745-751.

[9] Diederik P. Kingma and Jimmy Ba. 2015. Adam: A Method for Stochastic Optimization. In 3rd International Conference on Learning Representations, ICLR 2015 San Diego, CA, USA, May 7-9, 2015, Conference Track Proceedings, Yoshua Bengio and Yann LeCun (Eds.). http://arxiv.org/abs/1412.6980

[10] Bowen Li, Xiaojuan Qi, Thomas Lukasiewicz, and Philip HS Torr. 2020. Manigan: Text-guided image manipulation. In Proceedings of the IEEE/CVF Conference on Computer Vision and Pattern Recognition. 7880-7889.

[11] Jen-Yu Liu and Yi-Hsuan Yang. 2019. Dilated Convolution with Dilated GRU for Music Source Separation. In Proceedings of the Twenty-Eighth International foint Conference on Artificial Intelligence, IFCAI-19. International Joint Conferences on Artificial Intelligence Organization, 4718-4724. https://doi.org/10.24963/ijcai. 2019/655

[12] Yahui Liu, Marco De Nadai, Deng Cai, Huayang Li, Xavier Alameda-Pineda Nicu Sebe, and Bruno Lepri. 2020. Describe What to Change: A Text-guided Unsupervised Image-to-Image Translation Approach. In Proceedings of the 28th ACM International Conference on Multimedia. 1357-1365.

[13] Marco A Martínez Ramírez, Emmanouil Benetos, and Joshua D Reiss. 2020. Deep learning for black-box modeling of audio effects. APPLIED SCIENCES-BASEL 10, $2(2020)$.

[14] Marco A Martínez Ramírez and Joshua D Reiss. 2019. Modeling nonlinear audio effects with end-to-end deep neural networks. In ICASSP 2019-2019 IEEE International Conference on Acoustics, Speech and Signal Processing (ICASSP). IEEE, 171-175.

[15] Marco A Martínez Ramírez, Daniel Stoller, and David Moffat. 2021. A Deep Learning Approach to Intelligent Drum Mixing with the Wave-U-Net. Fournal of the Audio Engineering Society 69, 3 (2021), 142-151.

[16] Marco A Martínez Ramírez, Daniel Stoller, and David Moffat. 2021. A Deep Learning Approach to Intelligent Drum Mixing with the Wave-U-Net. fournal of the Audio Engineering Society 69, 3 (2021), 142-151.

[17] D. Matz, Estefanía Cano, and J. Abeßer. 2015. New Sonorities for Early Jazz Recordings Using Sound Source Separation and Automatic Mixing Tools. In ISMIR.
[18] Gabriel Meseguer-Brocal and Geoffroy Peeters. 2019. CONDITIONED-U-NET: Introducing a Control Mechanism in the U-net For Multiple Source Separations.. In 20th International Society for Music Information Retrieval Conference, ISMIR (Ed.).

[19] Stylianos Ioannis Mimilakis, Estefanıa Cano, Jakob Abeßer, and Gerald Schuller. 2016. New sonorities for jazz recordings: Separation and mixing using deep neural networks. In 2nd AES Workshop on Intelligent Music Production, Vol. 13.

[20] Jeffrey Pennington, Richard Socher, and Christopher D Manning. 2014. Glove: Global Vectors for Word Representation.. In EMNLP, Vol. 14. 1532-1543.

[21] Ethan Perez, Florian Strub, Harm de Vries, Vincent Dumoulin, and Aaron C Courville. 2018. FiLM: Visual Reasoning with a General Conditioning Layer. In AAAI.

[22] Zafar Rafii, Antoine Liutkus, Fabian-Robert Stöter, Stylianos Ioannis Mimilakis, and Rachel Bittner. 2017. MUSDB18 - a corpus for music separation. https://doi. org/10.5281/zenodo.1117371 MUSDB18: a corpus for music source separation.

[23] Martínez Ramírez and Joshua D Reiss. 2018. End-to-end equalization with convolutional neural networks. In 21st International Conference on Digital Audio Effects (DAFx-18).

[24] David Samuel, Aditya Ganeshan, and Jason Naradowsky. 2020. Meta-learning Extractors for Music Source Separation. In ICASSP 2020-2020 IEEE International Conference on Acoustics, Speech and Signal Processing (ICASSP). IEEE, 816-820.

[25] Mike Schuster and Kuldip K Paliwal. 1997. Bidirectional recurrent neural networks. IEEE transactions on Signal Processing 45, 11 (1997), 2673-2681.

[26] Michael Stein, Jakob Abeßer, Christian Dittmar, and Gerald Schuller. 2010. Automatic detection of audio effects in guitar and bass recordings. In Audio Engineering Society Convention 128. Audio Engineering Society.

[27] Christian J Steinmetz, Jordi Pons, Santiago Pascual, and Joan Serrà. 2020. Automatic multitrack mixing with a differentiable mixing console of neural audio effects. arXiv preprint arXiv:2010.10291 (2020).

[28] Christian J Steinmetz and Joshua D Reiss. 2021. Efficient Neural Networks for Real-time Analog Audio Effect Modeling. arXiv preprint arXiv:2102.06200 (2021).

[29] Stefan Uhlich, Marcello Porcu, Franck Giron, Michael Enenkl, Thomas Kemp, Naoya Takahashi, and Yuki Mitsufuji. 2017. Improving music source separation based on deep neural networks through data augmentation and network blending. In 2017 IEEE International Conference on Acoustics, Speech and Signal Processing (ICASSP). IEEE, 261-265.

[30] Ashish Vaswani, Noam Shazeer, Niki Parmar, Jakob Uszkoreit, Llion Jones, Aidan N Gomez, Łukasz Kaiser, and Illia Polosukhin. 2017. Attention is all you need. In Advances in neural information processing systems. 5998-6008.

[31] Emmanuel Vincent, Rémi Gribonval, and Cédric Févotte. 2006. Performance measurement in blind audio source separation. IEEE transactions on audio, speech, and language processing 14, 4 (2006), 1462-1469.

[32] Scott Wisdom, Efthymios Tzinis, Hakan Erdogan, Ron J. Weiss, Kevin Wilson, and John R. Hershey. 2020. Unsupervised Sound Separation Using Mixture Invariant Training. In NeurIPS. https://arxiv.org/pdf/2006.12701.pdf

[33] Alec Wright, Eero-Pekka Damskägg, Lauri Juvela, and Vesa Välimäki. 2020. RealTime Guitar Amplifier Emulation with Deep Learning. Applied Sciences 10, 3 (2020). https://doi.org/10.3390/app10030766

[34] Lonce Wyse. 2017. Audio spectrogram representations for processing with convolutional neural networks. arXiv preprint arXiv:1706.09559 (2017).

[35] Dacheng Yin, Chong Luo, Zhiwei Xiong, and Wenjun Zeng. 2019. PHASEN: A Phase-and-Harmonics-Aware Speech Enhancement Network. arXiv preprint arXiv:1911.04697 (2019).

[36] Jiahui Yu, Zhe Lin, Jimei Yang, Xiaohui Shen, Xin Lu, and Thomas S Huang. 2018. Generative image inpainting with contextual attention. In Proceedings of the IEEE conference on computer vision and pattern recognition. 5505-5514.

[37] Weitao Yuan, Shengbei Wang, Xiangrui Li, Masashi Unoki, and Wenwu Wang. 2019. A Skip Attention Mechanism for Monaural Singing Voice Separation. IEEE Signal Processing Letters 26, 10 (2019), 1481-1485.

[38] Jun-Yan Zhu, Taesung Park, Phillip Isola, and Alexei A Efros. 2017. Unpaired image-to-image translation using cycle-consistent adversarial networks. In Proceedings of the IEEE international conference on computer vision. 2223-2232. 